\title{
Aggregation of Single-phase Electric Vehicles for Frequency Control Provision Based on Unidirectional Charging
}

\author{
Sæmundsson, Valgeir Thor; Rezkalla, Michel M.N.; Zecchino, Antonio; Marinelli, Mattia
}

Published in:

Proceedings of the 52nd International Universities Power Engineering Conference

Link to article, DOI:

10.1109/UPEC.2017.8231867

Publication date:

2017

Document Version

Peer reviewed version

Link back to DTU Orbit

Citation (APA):

Sæmundsson, V. T., Rezkalla, M. M. N., Zecchino, A., \& Marinelli, M. (2017). Aggregation of Single-phase Electric Vehicles for Frequency Control Provision Based on Unidirectional Charging. In Proceedings of the 52nd International Universities Power Engineering Conference IEEE. https://doi.org/10.1109/UPEC.2017.8231867

\section{General rights}

Copyright and moral rights for the publications made accessible in the public portal are retained by the authors and/or other copyright owners and it is a condition of accessing publications that users recognise and abide by the legal requirements associated with these rights.

- Users may download and print one copy of any publication from the public portal for the purpose of private study or research.

- You may not further distribute the material or use it for any profit-making activity or commercial gain

- You may freely distribute the URL identifying the publication in the public portal 


\title{
Aggregation of Single-Phase Electric Vehicles for Frequency Control Provision Based on Unidirectional Charging
}

\author{
Valgeir Thor Sæmundsson, Michel Rezkalla, Antonio Zecchino, Mattia Marinelli \\ Center for Electric Power and Energy, Department of Electrical Engineering, \\ DTU - Technical University of Denmark, Roskilde, Denmark \\ vallisaem@gmail.com, mirez@elektro.dtu.dk, antozec@elektro.dtu.dk, matm@elektro.dtu.dk
}

\begin{abstract}
As the use of electric vehicles grows there is a greater possibility of using aggregated sets of electric vehicles as a large flexible unit to assist with the control of the power system. In this paper, the possibility of using electric vehicles as a flexible load for frequency control is investigated. The investigations are performed in a Pan-European interconnected grid with varying wind power penetration and different operational scenarios. Within this grid, the paper focuses on primary frequency control provision from electric vehicles and how the system behaves as the vehicles are being controlled within their respective areas. The investigations show that electric vehicles can be used for primary frequency control with different wind power penetration. By controlling the vehicles, the steady state frequency is improved and, since the vehicles react fast enough to the frequency changes, also frequency nadir and rate of change of frequency are positively affected.
\end{abstract}

Index Terms -- Electric vehicles; Frequency control; Power system stability; Power system modeling.

\section{INTRODUCTION}

The electrical energy generation is changing from centralized generation units to decentralized generation units with the introduction of renewable energy sources (RES). The transition to a decentralized, fossil-free energy system has to a certain degree caused the system controllers to lose control of the energy generation in the system. The production from the decentralized units is characterized by stochasticity, e.g. due to the weather dependency. In addition, the deployment of a large amount of renewable energy sources into the power system causes a loss of inertia in the system. Current renewable energy sources are connected to the grid through power converters; therefore they do not provide any mechanical inertia contribution to the system. This means that the system becomes more sensitive to all power imbalances, which can cause significant frequency changes, and in the worst case, a blackout of the system [1]-[4].

The requirement of ensuring system balance is therefore becoming more challenging, compared to the traditional power system which included large centralized generation units. The large centralized generation units were easy to control for maintaining the systems power balance and provided a large inertial response in the system due to the large synchronous rotating mass of the conventional units. These units are now gradually being shut down and replaced by RES. The system operators are therefore looking for new ways to ensure the system stability [5].

With the current growth in the number of electric vehicles (EVs) comes the opportunity of using the vehicles to maintain the power balance in the system, as the EVs can be considered as energy storage systems. If the charging of the EVs could be adequately controlled, a great control possibility could be achieved. By using the EVs, the control of the system would be moved - by a certain degree - from controlling the generation to controlling the consumption [6]-[10].

Ancillary services represent an important part of the operational costs of networks and are critical for an efficient and secure grid operation. Typical examples are active power balancing (frequency control and stability, in which the EVs could contribute to), voltage control and stability, transmission system security and black start capability. Denmark is a part of two synchronous power systems with different requirements for the ancillary services which the EVs controllers must fulfill.

The aim of the paper is to implement and simulate sets of aggregated EVs in a high voltage grid, to investigate the possibility of using the EVs to function as ancillary services (frequency control and stability). The study has been carried out by means of RMS simulation activities in DIgSILENT PowerFactory software environment. The paper is structured as follows. Section II presents the modelled grid. Primary frequency control by EVs is reported in Section III, together with a detailed description of the proposed innovative controller. Section IV presents the simulation studies: the scenarios are defined, and results are presented and discussed.

\section{SYSTEM LAYOUT}

The modelled grid is called the Pan-European grid and is used for research purposes in the European project ELECTRA IRP [11], [12]. The grid is built up to resemble the European transmission grid with its transmission voltages and a grid frequency of $50 \mathrm{~Hz}$. The transmission voltages in the PanEuropean grid are $220 \mathrm{kV}$ and $400 \mathrm{kV}$ just as in the European transmission system. The generation units are set at $20 \mathrm{kV}$ buses where step-up transformers connect to the main grid while the loads are connected directly to the main grids buses [13]. 
The Pan-European grid is built up by four areas or cells which are then connected by transmission lines of varying lengths. Three of the areas are AC grids while the fourth is a DC part. In order not to overcomplicate the simulations study it was decided not to include the DC wind park which is in the DC part of the Pan-European grid. Nevertheless, the DC converters play a critical role in the voltage control of the system and therefore it was decided to use the converters for voltage regulation.

The three AC grid areas include a different amount of the conventional and wind power generation as well as load. Area 1 has the biggest share of the nominal generation, while Area 2 has no wind power generation while it is the area with the largest share of the load. Area 2 plays an important role in the simulations as the reference bus and slack machines are placed there.

The Pan-European grid includes:

- 9 conventional synchronous machines (500 MVA each)

- 410 wind turbines (6.7 MVA each)

- Several 3 phase loads with a total demand of $2600 \mathrm{MW}$ and power factor of 0.95

- $\quad$ The total system inertia $(2 \mathrm{H})$ spans from 10 seconds when no wind turbines are connected to 2.5 seconds in the $90 \%$ penetration scenario.

The EVs are modelled as single-phase loads which are located at a residential voltage level. The EVs are implemented with a controller which can control the charging level of the vehicles. The controller is programmed to allow the EVs to provide fast primary control. The control process and the EVs capability to provide frequency control is tested on several operations scenarios. The control process includes some time delays which affect the EVs reactions to a frequency change [14].

\section{Modeling THE AGGREGATED SET OF EVS}

In this paper it is assumed that $42 \%$ of all personal vehicles in Denmark will be electric and $10 \%$ of them $(100,000 \mathrm{EVs})$ can be used or the frequency control at any given time. The Pan-European grid includes roughly half of the average load in Denmark, to have to comparison to the Danish grid it was decided to include only 50,000 EVs in the modelled grid. It was decided to place the EVs at three buses, one bus in each of the three AC grid areas within the Pan-European grid. The EVs are aggregated in groups of 1111 vehicles which are represented as one single-phase load as indicated in Fig. 1. The single-phase loads are then placed in sets of five on each phase on the three buses. This is done to have the possibility of simulating different EVs and controller characteristics on the same bus. The $1111 \mathrm{EVs}$ are to be aggregated together and are therefore equipped with a controller. Each of the singlephase loads has a controller which measures the frequency at the load's terminals and controls the active power consumption of the load (which is the charging of the EVs).

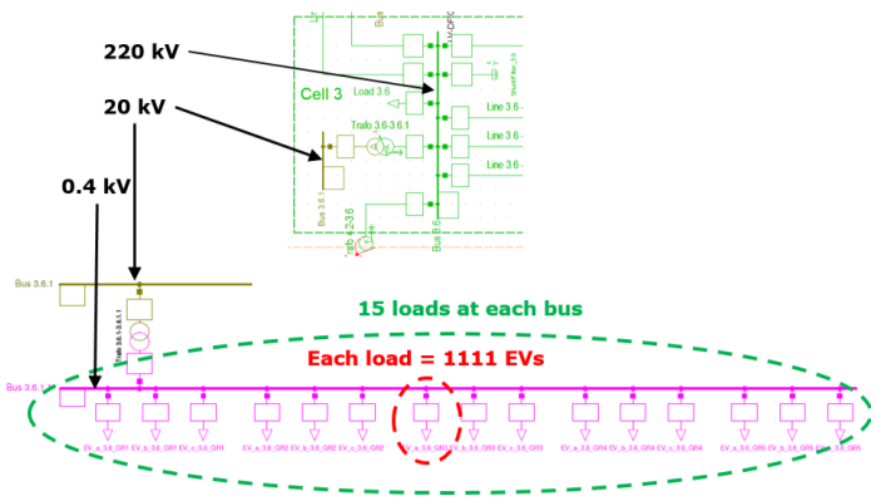

Fig. 1. Implementation of the EVs into the simulations grid.

\section{A. Aggregation method}

The EVs are controlled by changing the charging current in the range of 6-16 $\mathrm{A}$, as this is the charging range given by the standard IEC61851. This means that in normal operation where the frequency is $50 \mathrm{~Hz}$, the vehicles will be charging at $11 \mathrm{~A}$, meaning that they can offer a symmetrical flexibility window for up/down-frequency regulation [10]. The EVs do not participate in the voltage control and do therefore not consume or generate any reactive power. Each single-phase load is given the size of $2.8 \mathrm{MW}$ and the load type of $1 \mathrm{PH}-$ $\mathrm{PH}-\mathrm{N}$ as it is a single-phase load that uses the neutral.

The vehicles were grouped into 45 different single-phase loads. Each load has its own controller which includes the EV and controller characteristics reported in Fig. 2. Therefore, it is possible to simulate the different time delays caused by the controller or the EV itself. The time delays represent the time needed for the measurement, the set-point calculation, and the communication.

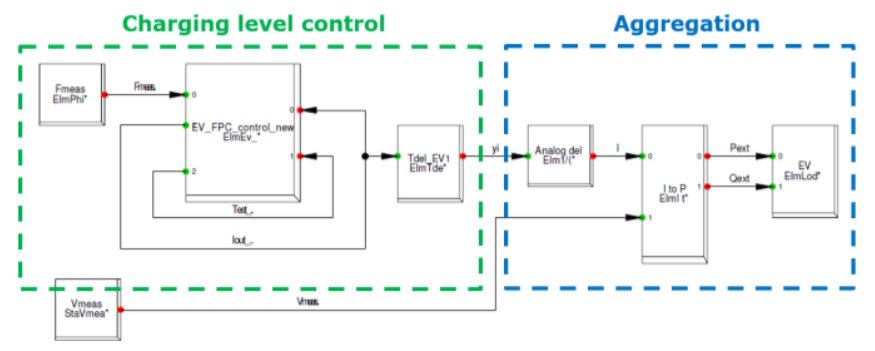

Fig. 2. The controller structure in PowerFactory.

\section{B. EV Control}

The controller measures the voltage and frequency at the $0.4 \mathrm{kV}$ bus, which the respective vehicles are connected to, by the Fmeas and Vmeas blocks (see Fig. 2). The EVs do not participate in voltage control, but the voltage measurement signal is connected to the $I$ to $P$ block to create a linear voltage dependency. The EVs reactive power is set to zero since they are not participating in the voltage control.

The measured frequency signal is connected to the EV_FPC_control_new block (see Fig. 3) where the frequency signal is changed to a current signal. This is performed as the desired regulation is to have the charging current of the vehicles reacting to changes in the frequency. The 
functionality of the $E V \_F P C \_c o n t r o l \_n e w$ block is described later in this section.

By looking back at diagram described in Fig. 2, it can be noticed that the current signal from the $E V \_F P C \_c o n t r o l \_n e w$ block is connected to the Tdel_EV, giving the time delay of the EVs, which represents the time requested from the EVs to get activated. The delayed current signal is then connected to the Analog del block which changes the stepped current signal to an exponential behavior. This is done because each of the loads represents a number of EVs instead of just one. That means that not all the vehicles will react in the same way to the controllers' set-point, as each vehicle has its own characteristic.

The purpose of the last two blocks in Fig. 2 is to get the signal from the controller to the single-phase loads as each load represents many vehicles. The $I$ to $P$ block receives the measured voltage signal and the current signal from the $T d e l \_E V$ block. The block has embedded equations to change the current signal into an active power signal while, as mentioned earlier, the reactive power signal is set to zero. Each single-phase load active power $\left(\mathrm{P}_{\text {nom }}\right)$ is $2.8 \mathrm{MW}$ at normal operation, where the charging current $\left(\mathrm{I}_{\text {nom }}\right)$ is $11 \mathrm{~A}$. The active power has to change with the current to enable the frequency control.

$$
\begin{aligned}
& P_{\text {ref }}=I \cdot \frac{P_{\text {nom }}}{I_{\text {nom }}} \\
& Q_{\text {ext }}=0
\end{aligned}
$$

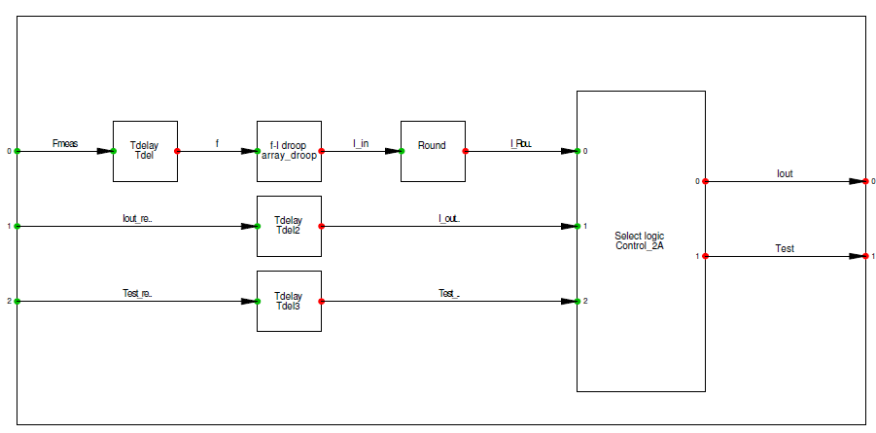

Fig. 3. The EV_FPC_control_new block structure in PowerFactory.

The EV block receives the calculated active power signal and fixed reactive power signal. The block represents the single-phase load connected to the controller. The singlephase load represents the aggregated vehicles which receives the signal from the block. Droop characteristic

The EV_FPC_control_new block (see Fig. 3) is where the measured frequency signal is changed to a current signal, which is then used further by the controller. The block takes the measured frequency signal into the block Tdelay which adds a measurements time delay to the signal. The delayed frequency signal is then connected to the $f$-I droop block which alters the frequency signal to a current signal by usage of the droop curve reported in Fig. 4.

\section{The droop characteristics}

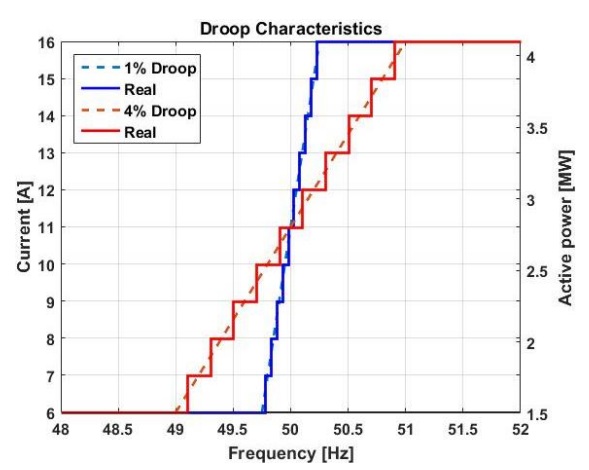

Fig. 4. The droop characteristics for the controller.

The current signal from the $f$-I droop block (see Fig. 3 ) is connected to the Round block which rounds the current signal to the nearest integer number. This process is performed due to a requirement set by the IEC61851 standard as was considered in [13]. The rounded current signal is then connected to the Select logic block which reduces possible oscillations of the rounded current signal. If the current signal from the $f$-I droop block is in the middle of two consecutive integer numbers and changing slightly, it would cause undesired oscillations of the EVs charging current. To avoid this the block is programmed in such a way that the charging current can change one ampere in one direction, if the control signal wants to change the direction, the controller will need a signal of a two ampere change of the current. To achieve this, the Select logic block needs to have the former current value and direction of the current change. To make this possible in the program the two Tdelay blocks are used, which have the time delays of Tdel2 and Tdel3. The delayed signals I_out. and Test_ are connected to the Select logic block which will use them to reduce the oscillations as much as possible by comparing the new signal to the old one. The old current signal will include the old current value while the test signal indicates the direction of the current change. The current change direction will be given by $1,0,1$, where -1 is for a decreasing current, 1 for increasing current and the 0 is for the initialization of the controller.

How the algorithm is constructed and how each variable is taken into account is described in [13]. How the frequency signal is processed and affected by the time delays can be seen in the example reported in Fig. 5.

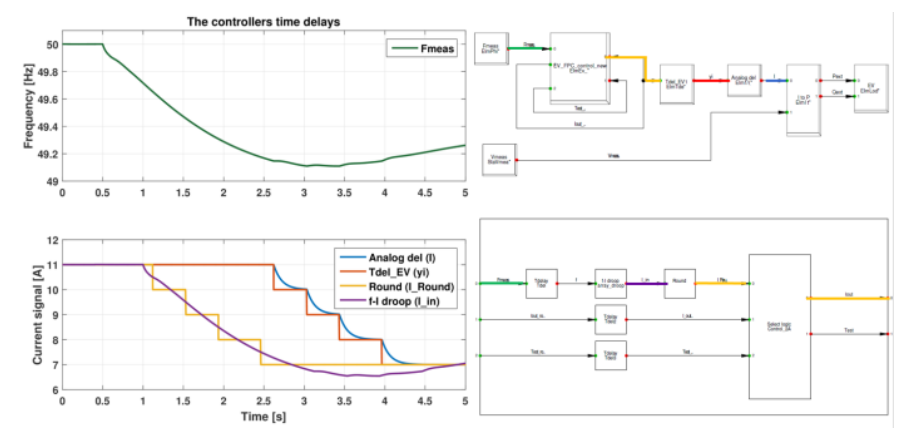

Fig. 5. The control process by the controller. 


\section{SimULATIONS SCENARIOS AND RESUlTS}

All the simulation scenarios include the same event in the grid, which entails one of the generators falling out of service. The generator has a fixed active power generation while the reactive power depends on the voltage, as the machine contributes to the voltage control. The machines active power generation is $5 \%$ of the grids total load (that is, the three-phase loads and all the EVs at a charging current of $11 \mathrm{~A}$ ).

Table 1 shows the simulations scenarios and cases, and which operation cases will be investigated. The first three scenarios are performed to investigate the participation of the EVs. The system is tested with and without controlling the EVs in the grid with a different amount of wind power penetration. After having tested the control process of the EVs, the droop characteristics of the EVs controller can be tested in a grid with a high wind power penetration. The droop characteristics of the EVs controller show how sensitive the vehicles are for changes in the frequency. A low droop value could therefore cause oscillations in the charging current. The droop characteristics are therefore tested with and without the logic part in the controller.

Scenarios 5 and 6 are performed to determine how the system reacts if the EVs do not have the same response to the set-point given by the controller. The controllers in the three areas can have different time delays and therefore cause different delays between the three areas. The grid can include different controllers and/or EVs at the buses which can cause different time delays between the phases. The last scenario (Scenario 7) is performed to investigate how the system performs if the control process includes lower time delays.

TABLE 1

Overview of the simulations scenarios and cases.

\begin{tabular}{|c|c|c|c|c|c|}
\hline Scenario & Case & EVs control droop & Logic Block & Time delays & RES \\
\hline \multirow{2}{*}{1} & A & OFF & ON & Same & $0 \%$ \\
\cline { 2 - 6 } & B & $4 \%$ & ON & Same & $0 \%$ \\
\hline \multirow{2}{*}{2} & A & OFF & ON & Same & $25 \%$ \\
\cline { 2 - 6 } & B & $4 \%$ & ON & Same & $25 \%$ \\
\hline \multirow{2}{*}{3} & A & OFF & ON & Same & $90 \%$ \\
\cline { 2 - 6 } & B & $4 \%$ & ON & Same & $90 \%$ \\
\hline \multirow{4}{*}{4} & A.a & $4 \%$ & ON & Same & $90 \%$ \\
\cline { 2 - 6 } & A.b & $4 \%$ & OFF & Same & $90 \%$ \\
\cline { 2 - 6 } & B.a & $1 \%$ & ON & Same & $90 \%$ \\
\cline { 2 - 6 } & B.b & $1 \%$ & OFF & Same & $90 \%$ \\
\hline \multirow{5}{*}{$\mathbf{n}$} & -- & $1 \%$ & ON & Different between areas & $90 \%$ \\
\hline $\mathbf{6}$ & - & $1 \%$ & ON & Different between phases & $90 \%$ \\
\hline $\mathbf{7}$ & -- & $1 \%$ & ON & Different & $90 \%$ \\
\hline
\end{tabular}

\section{A. Scenarios 1-3: The participation of the electric vehicles}

The simulations are performed to investigate how the EVs are reacting in the grid with different levels of wind power penetration. The EVs are simulated without any control and with a $4 \%$ droop characteristics for the frequency control. In the case where the vehicles are not controlled they act as a constant load (see from Fig. 6 and Fig. 7).

The scenarios showed that the EVs can contribute to the frequency control and do not have any noticeable negative effects on the system. It was noticed from the simulations that the vehicles have no effect on the nadir or the ROCOF values.
This is because the vehicles are simulated with some time delays within the control process. The governors of the machines are therefore faster to make the frequency increase than the vehicles can start to change their active power consumption. However, the EVs do raise the steady state value of the frequency if the control process is activated and thereby assist with the frequency control.
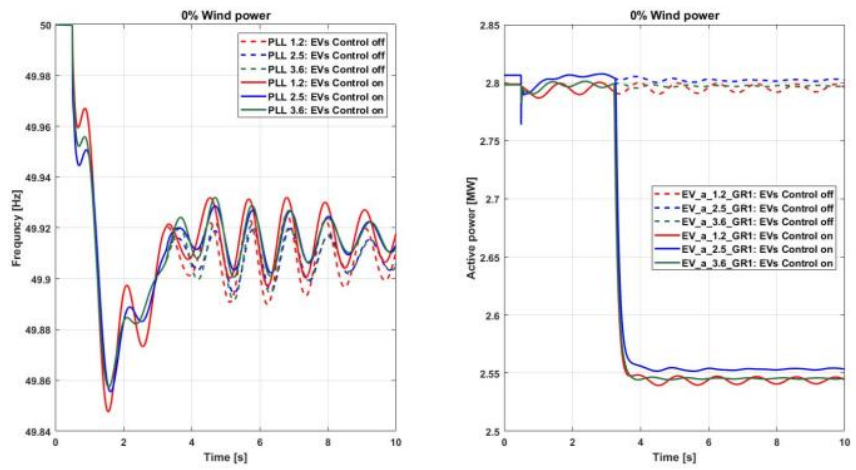

Fig. 6. The EVs buses frequencies and active power consumption at $0 \%$ wind power penetration.
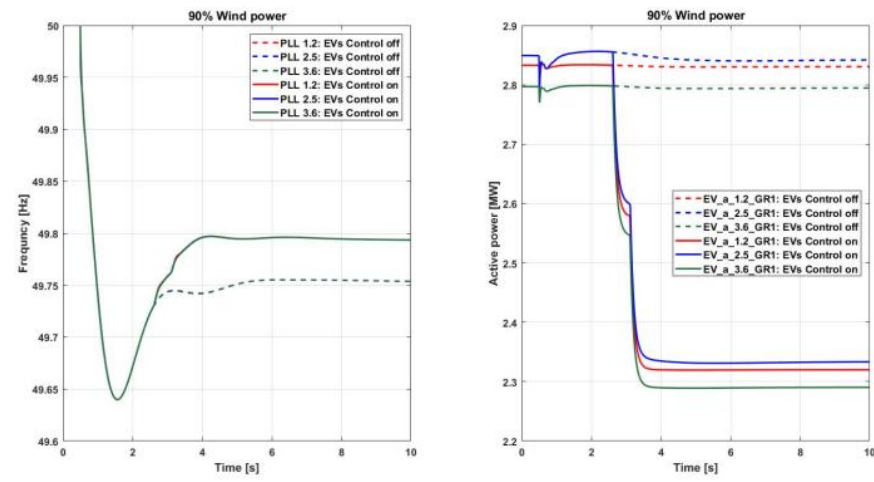

Fig. 7. The EVs buses frequencies and active power consumption at $90 \%$ wind power penetration.

\section{B. Scenario 4: The droop characteristics}

The investigation is performed to investigate how the EVs react and affect the system when the controller is given a lower droop value. The investigation is performed on a grid with a $90 \%$ wind power penetration, where there are only two conventional generators active after the event. The controller is simulated with a $4 \%$ droop as in scenarios 1-3 and with a $1 \%$ droop characteristics for the frequency control both with and without the logic part.

The Logic part was tested in the scenario and was proven to be an important part of the control process. The frequency was stable, had a higher steady-state value and the number of changes of the charging level was reduced if the Logic part was activated. If the controller was tested with a $1 \%$ droop characteristic, the control process began to oscillate when the logic part was deactivated as reported in Fig. 8. 


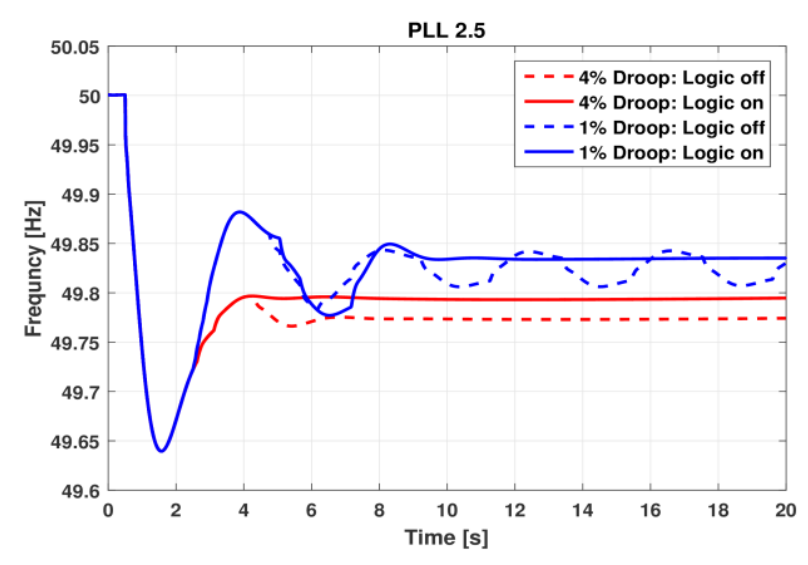

Fig. 8. The frequency at bus 2.5 with different droop values.

\section{Scenario 5: Different time delays between areas}

The simulation is performed to investigate how different control process time delays between the areas affect the system. The different time delays used for the investigation can be found in TABLE 2. The investigation is performed on the same grid as in scenario 4 .

TABLE 2

The different time delays within the areas.

\begin{tabular}{|l|c|c|c|}
\hline \multicolumn{4}{|c|}{ Tdel_EV } \\
\hline & Area 1 [s] & Area 2 [s] & Area 3 [s] \\
\hline Case 1 & 1.5 & 1.5 & 1.5 \\
\hline Case 2 & 1.5 & 1.0 & 1.0 \\
\hline Case 3 & 1.5 & 0.5 & 1.0 \\
\hline Case 4 & 2.5 & 1.5 & 2.0 \\
\hline Case 5 & 6.5 & 0.5 & 4.0 \\
\hline
\end{tabular}

The simulations results reported in Fig. 9 show that the nadir value is not the same for all the cases but it was noticed that the cases with a higher nadir have a lower steady-state frequency than the other cases.

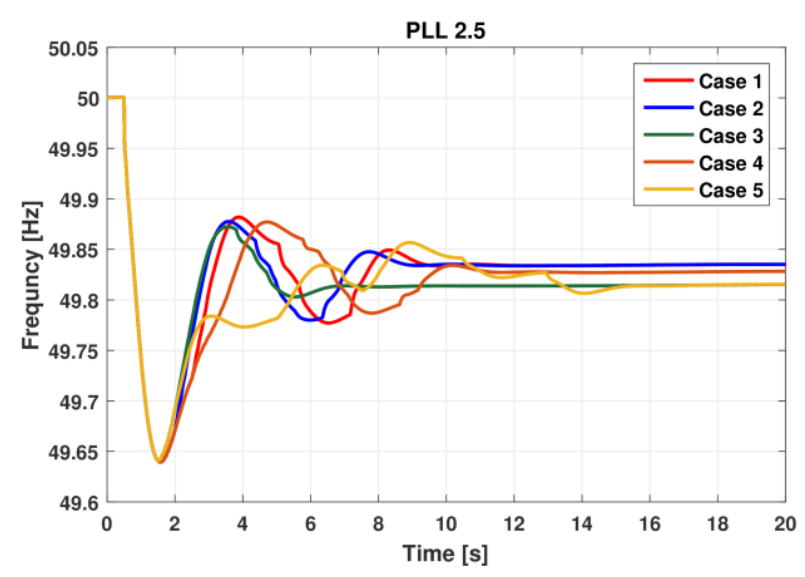

Fig. 9. The frequency at bus 2.5 with different time delays in the areas.

This occurs because the higher nadir causes a lower overshoot of the frequency, which is the frequency peak after the nadir. This means that the charging level would only fall one level in cases 3 and 5, but is prevented by the logic part. The other three cases include the extra step in the charging level and therefore the charging level change is allowed by the logic part as the change is two steps. This results in a higher charging level in cases 3 and 5, leading to a lower steady-state frequency.

\section{Scenario 6: Different time delays among phases}

The simulation is performed to investigate how a different distribution of the EVs on the three phases affects the system. The vehicles are distributed on the phases by giving different time delays to the vehicles on the three phases. The investigation is performed on the same grid as in scenarios 45. Results show that the steady-state frequency is higher in case 3 than in case 2. As explained before this is because the active power consumption of the EVs is changed only once and that is to the lowest consumption possible for the vehicles. The load unbalance between the phases does not seem to have a negative influence on the frequency or the vehicles control process. The voltage unbalance factor does not exceed the $2 \%$ limit at any time of the simulations period and therefore it can be concluded that the EVs control process does not cause a larger voltage unbalance than the standards allow.

\section{E. Scenario 7: Fast-reacting vehicles}

The investigation is performed to investigate what advantages and disadvantages come with shorter time delays within the control process of the EVs. The investigation is performed on the same grid as in scenario 4, 5 and 6.

TABLE 3

The delays in the different cases.

\begin{tabular}{|c|c|c|c|}
\hline \multicolumn{5}{|c|}{ Time delays } \\
\hline & Tdel_EV [s] & Tdel [s] & T [s] \\
\hline Case 1 & 1.5 & 0.5 & 0.1 \\
\hline Case 2 & 0.1 & 0.1 & 0.1 \\
\hline Case 3 & 0.01 & 0.01 & 0.01 \\
\hline
\end{tabular}

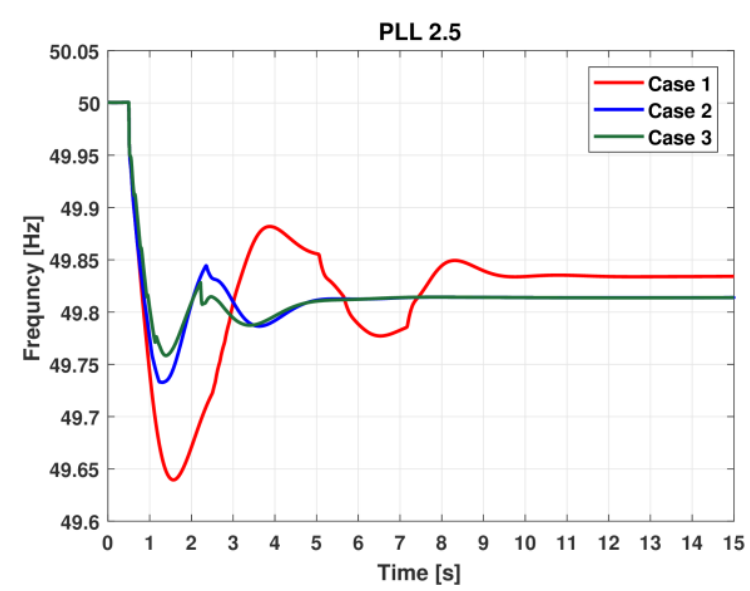

Fig. 10. The frequency at bus 2.5 with different delays in the EVs model.

The simulations performed in the previous scenarios showed that the ROCOF values always remained the same. The investigation performed in scenario five showed the nadir is improved with fast reaction from the EVs. It was found through the simulations in scenario 7 that the ROCOF value 
changed through the cases where the time delays of the control process were reduced as reported in Fig. 10.

\section{CONCLUSION AND FUTURE WORK}

The possibility of using the EVs for primary frequency control was investigated on the Pan-European grid with different levels of wind power penetration. Unidirectional single-phase charging vehicles were successfully aggregated within their respective area. The simulations scenarios showed that the aggregated EVs can contribute to the frequency control by raising the steady-state frequency and do not have any noticeable negative effects on the system. The different levels of wind power penetration did not affect the EVs much but with $90 \%$ wind power penetration a greater reaction was observed from the vehicles because the frequency fell to a lower level than in the other scenarios. The EVs controller was first simulated with a $4 \%$ droop characteristic but it was found that a $1 \%$ droop gave a better result if the logic part was activated. With a $1 \%$ droop characteristic for the controller the logic part is necessary to prevent oscillations within the control process as the control process becomes more sensitive for any frequency changes. The control process includes some time delays which represent all the different time delays of the whole control process. Those time delays were used in the investigations as the different areas and vehicles might not have the same characteristics. By giving different time delays to the controllers in the three areas the vehicles had different reactions times among the areas. This was found to cause no instabilities or significant behavior changes in the grid. In two of the cases the EVs in one area were given a fast reaction time and it was noticed that there was a higher value in the nadir but a lower steady-state frequency. When the grid was tested with different time delays between the phases no instabilities were found while some imbalances were noticed in the voltage. Again two cases which included fast reacting vehicles and the same changes were noticed in the nadir and steady-state frequency. The different time delays between the area and the phases do not cause any instability but different time delays among the phases do cause imbalances within the grid, which can be seen in the grids currents and voltage levels as well as the low voltage transformers neutral current. The last scenario was performed to investigate if the vehicles could change the ROCOF value by reacting fast to the frequency changes. The controllers were given very low time delays values for this investigation. The investigation showed that the fast reacting vehicles affected the ROCOF and nadir value, but as previous investigations had shown, with a lower steadystate frequency.

Future work will investigate possible conflicts between frequency control and voltage control by electric vehicles as well as assessing how often there is a need in the power system for upward regulation reserve in the primary frequency control in order to better size the initial charging current of a set of EVs participating in frequency control based on unidirectional charging.

\section{ACKNOWLEDGEMENTS}

The work in this paper has been partly supported by the European FP7 project ELECTRA (grant no: 609687; web site www.ElectraIRP.eu) and partly by the Danish research project Electra Top-up (grant: 3594756936313).

\section{REFERENCES}

[1] B. Kroposki et al., "Achieving a 100\% Renewable Grid: Operating Electric Power Systems with Extremely High Levels of Variable Renewable Energy," in IEEE Power and Energy Magazine, vol. 15, no. 2, pp. 61-73, March-April 2017.

[2] P. Tielens and D. Van Hertem, "The relevance of inertia in power systems," Renew. Sustain. Energy Rev., vol. 55, pp. 999-1009, 2016

[3] European Network of Transmission System Operators for Electricity (ENTSO-E), "Nordic Balancing Philosophy," tech. rep., June 2016.

[4] European Network of Transmission System Operators for Electricity (ENTSO-E), "P1 - Policy 1: Load-Frequency Control and Performance," tech. rep. [Online]. Available: http://www.entsoe.eu.

[5] DGA Consulting, "International review of frequency control adaptation," tech. rep. [Online]. Available: https://www.aemo.com.au

[6] P. M. R. Almeida, F. J. Soares, and J. A. P. Lopes, "Electric vehicles contribution for frequency control with inertial emulation," Electric Power Systems Research, vol. 127, pp. 141-150, 2015.

[7] S. You, J. Hu, C. Ziras, “An Overview of Modeling Approaches Applied to Aggregation-Based Fleet Management and Integration of Plug-in Electric Vehicles $\dagger$," Energies 2016, 9, 968.

[8] K. Knezović, S. Martinenas, P. B. Andersen, A. Zecchino, and M. Marinelli, "Enhancing the role of EVs in the grid: field validation of multiple ancillary services provision," Transportation Electrification, IEEE Transactions on, vol. 3, no. 1, pp. 201-209, Mar. 2017.

[9] J. Hu, H. Morais, T. Sousa, M. Lind, "Electric vehicle fleet management in smart grids: A review of services, optimization and control aspects, Renewable and Sustainable Energy Reviews," Volume 56, April 2016 , Pages 1207-1226,

[10] M. Marinelli, S. Martinenas, K. Knezović, and P. B. Andersen, "Validating a centralized approach to primary frequency control with series-produced electric vehicles," J. of Energy Storage, vol. 7, pp.63-73, Aug. 2016.

[11] L. Martini, A. Morch, L. Radaelli, C. Caerts, C. Tornelli, S. Hänninen, and H. Brunner, "Electra IRP approach to voltage and frequency control for future power systems with high DER penetration," in $23 \mathrm{rd}$ International Conference on Electricity Distribution CIRED, 2015, pp. 1-4, Lyon, 15-18 June 2015

[12] K. Visscher, M. Marinelli, A.Z. Morch, and S.H. Jakobsen, "Identification of observables for future grids - The framework developed in the ELECTRA project," In PowerTech, 2015 IEEE Eindhoven, pp.1-6, June 29 2015-July 22015.

[13] M. Marinelli, M. Pertl, M. Rezkalla, M. Kosmecki, S. Canevese, A Obushevs, A. Morch, "The Pan-European Reference Grid Developed in the ELECTRA Project for Deriving Innovative Observability Concepts in the Web-of-Cells Framework," Universities Power Engineering Conference (UPEC), 2016 Proceedings of the $51^{\text {st }}$ International, pp. 1-6, Coimbra, 6-9 Sep. 2016

[14] A. Zecchino, M. Rezkalla, M. Marinelli, "Grid Frequency Support by Single-Phase Electric Vehicles: Fast Primary Control Enhanced by a Stabilizer Algorithm," Universities Power Engineering Conference (UPEC), 2016 Proceedings of the 51st International, pp. 1-6, Coimbra, 6-9 Sep. 2016. 